\title{
Perfil de usuários de psicofármacos atendidos em uma farmácia comunitária do alto sertão paraibano
}

\section{Profile of psychotropic drug users treated in a Community Pharmacy of Upper Paraíba backwoods.}

\author{
Nathalia Minelli Medeiros de Sousa \\ Especialista em Farmácia Clínica pelo Instituto de Pós-Graduação de Goiás (IPOG) Cajazeiras - PB, Brasil. E-mail: \\ nathalia-minelli@hotamil.com. \\ Natália Bitú Pinto \\ Doutora em Farmacologia, Universidade Federal do Ceará (UFC). Professora da Faculdade Santa Maria (FSM) \\ Cajazeiras - PB, Brasil. E-mail: nataliabitu@gmail.com \\ Francileuda Batista de Almeida \\ Graduada em Farmácia, Faculdade Santa Maria (FSM). E-mail: farmaciasantamariacz@ gmail.com. \\ Neurislene da Silva Maciel \\ Acadêmica do Curso de Bacharelado em Farmácia, Faculdade São Francisco da Paraíba (FASP) Cajazeiras - PB, \\ Brasil. E-mail: neurislene2@gmail.com \\ Symara Abrantes Albuquerque de Oliveira Cabral \\ Discente do Mestrado em Sistemas Agroindustriais da Universidade Federal de Campina Grande - Pombal - PB, \\ Brasil. E-mail: symara_abrantes@ @otmail.com \\ Maria Carmem Batista de Alencar \\ Discente do Mestrado em Sistemas Agroindustriais da Universidade Federal de Campina Grande - Pombal - PB, \\ Brasil. E-mail: carmemsjp@ hotmail.com
}

\begin{abstract}
RESUMO: O estudo teve o objetivo de identificar o perfil dos usuários de psicofármacos atendidos em uma farmácia comunitária no município de Cajazeiras - PB. A pesquisa é do tipo exploratória descritiva, com abordagem quantitativa. A coleta de dados foi realizada em agosto a outubro de 2011, com uma entrevista estruturada aplicada a uma amostra de140 clientes. Os resultados mostram que $79 \%$ da população estudada foram mulheres, apresentando idade entre $40 \mathrm{e}$ 49 anos. O Clonazepam foi o medicamento mais consumido; $40 \%$ utilizavam a mais de um ano; $94 \%$ relataram ter tido melhoras no seu quadro clínico após o uso; $42 \%$ afirmaram usar esse medicamento para tratamento de insônia; 52\% responderam que fazem uso de outros medicamentos concomitantemente ao tratamento com psicofármacos, o que é um dado importante devido às interações medicamentosas. Assim, esta havendo um aumento da incidência de transtornos psiquiátricos, o que é um problema de saúde pública, gerando um impacto social e econômico. Dessa forma, é importante que o farmacêutico oriente os pacientes com relação ao uso de psicofármacos, a fim de evitar efeitos tóxicos e interações medicamentosas, e buscando aumentar a eficácia do tratamento e reduzindo a falta de adesão ao tratamento.
\end{abstract}

Palavras-chaves: Sistema Nervoso Central, Psicofármacos, Farmácia Comunitária.

ABSTRACT: The study aimed to identify the profile of psychotropic drug users seen at a community pharmacy in the city of Cajazeiras - PB. The research is descriptive exploratory with a quantitative approach. Data collection was conducted in August-October 2011, with a structured interview applied to a sample of 140 customers. The results show that $79 \%$ of the population were women, with ages ranging from 40 to 49 years. The Clonazepam was the most consumed drug; $40 \%$ used for more than one year; $94 \%$ reported having had improvements in their clinical condition after use; $42 \%$ said they use this medication to treat insomnia; $52 \%$ answered that they use other drugs concomitantly to treatment with psychotropic drugs, which is an important data due to drug interactions. Thus, this having an increased incidence of psychiatric disorders, which is a public health problem, generating a social and economic impact. Thus, it is important that the east pharmacist patients regarding the use of psychotropic drugs in order to avoid toxic effects and drug interactions, and seeking to increase the effectiveness of treatment and reduce the lack of adherence to treatment.

Key words: Central Nervous System, Pharmacotherapy, Community Pharmacy . 


\section{INTRODUÇÃO}

O surgimento dos psicofármacos causou uma vasta reformulação no campo da psiquiatria, com mudanças radicais nas perspectivas dos transtornos mentais, diminuindo a quantidade e o tempo de pacientes hospitalizados e devolvendo a sua qualidade de vida.

Os transtornos mentais mais freqüentes na população em geral são os psicóticos, os depressivos, os de ansiedade e os ocasionados por doenças neurodegenerativas que são mais freqüentes na fase senil (DUCAN; SCHMIDT; GIUGLIANI, 2004).

Alves (1994), estimou que os transtornos mentais apresentam uma prevalência de $20 \%$ na população brasileira. A partir de pesquisas epidemiológicas realizadas em cidades brasileiras encontrou-se resultados de buscas por cuidados psiquiátricos que variam de $34 \%$ em Porto Alegre e Brasília e 19\% em São Paulo.

Os principais psicofármacos em uso na atualidade: os antidepressivos, os ansiolíticos e sedativos, os antipsicóticos ou neurolépticos, os anticonvulsivantes, os opióides, os estimulantes psicomotores, os antiparkisonianos e os antialzheimer. São medicamentos que agem especialmente no sistema nervoso central (SNC) estimulando ou inibindo a ação dos neurotransmissores (RANG et al., 2007).

Apesar das legislações vigentes que regulamenta o uso de medicamentos de controle especial, o uso abusivo continua em grande escala, isso devido à exacerbação das prescrições ou a livre aquisição de maneira ilegal em estabelecimentos como farmácias e drogarias, ou até mesmo na internet, o que se tornou uma prática culturalmente arraigada na população brasileira (NOTO et al., 2002).

O uso de psicofármacos abordado durante o estudo é considerado elevado o que pode ocasionar dependência física e psicológica, por ter ação farmacológica principalmente no sistema nervoso central (SNC). Sendo assim, quais os psicofármacos mais dispensados na farmácia comunitária? E qual o perfil dos usuários de psicofármacos?

Diante desta problemática, surgiu o interesse em realizar este estudo, tendo por finalidade melhor compreender os problemas relacionados ao uso de psicofármacos. Espera-se que este estudo possa contribuir para direcionar práticas assistenciais aos usuários de psicofármacos na busca por utilização racional e segura, proporcionando um tratamento farmacológico eficaz e melhorando a qualidade de vida dos pacientes.

\section{MATERIAL E MÉTODOS}

Trata-se de um estudo exploratório descritivo, com uma abordagem quantitativa, realizada em uma Farmácia Comunitária localizada no município de Cajazeiras, no alto sertão paraibano, no extremo Oeste do estado, possuindo altitude de 298 metros no nível do mar, distante $477 \mathrm{~km}$ da capital João Pessoa, com uma área territorial de $586.275 \mathrm{Km}^{2}$. Com uma população estimada de 58.319 habitantes, sendo considerada a sexta maior cidade da Paraíba (BRASIL, 2010).
A Farmácia dispõe de um Farmacêutico, dois Gerentes Administrativos, uma Técnica em Enfermagem e quatro Balconistas. Com funcionamento de 24h00min durante todos os dias da semana, e tem suas atividades estabelecidas de acordo com as boas práticas farmacêuticas.

A coleta de dados foi realizada por entrevista, com roteiro estruturado, em agosto a outubro de 2011, após parecer favorável o Comitê de Ética da Faculdade Santa Maria.

Participaram da amostra 140 clientes atendidos na farmácia, os quais atenderam aos critérios de inclusão e concordaram mediante a assinatura do Termo de Consentimento Livre e Esclarecido (TCLE) participar do estudo. As informações coletadas nas entrevistas foram manuscritas no próprio roteiro e submetidas à organização e análise dos dados. O instrumento de coleta de dados continha as seguintes seções:

Secão A: para verificar às seguintes variáveis identificação (gênero, idade, raça/cor e situação conjugal) e perfil social (escolaridade e atividade profissional atual).

Secão B: questões referentes ao estilo de vida ingestão de bebidas alcoólicas.

Secão C: questões referentes à terapia medicamentosa - tempo de uso do medicamento, os principais sintomas para está fazendo uso desse medicamento, qual o medicamento (princípio ativo, nome comercial, dose e posologia), se recebeu orientação sobre os efeitos ou riscos do medicamento, com que frequiência utiliza-o, se teve reações desagradáveis, se alguém que reside na mesma casa utiliza o mesmo medicamento, se faz uso de outros medicamentos e de terapias alternativas para o tratamento da doença ou transtorno do sistema nervoso.

Para o processamento dos dados foi realizada um análise quantitativa, com a consolidação das informações, viabilizando caracterizar os indivíduos de nossa amostra. Os dados foram expressos em tabelas e gráficos, construídos no Programa Microsoft Excel 2010, posteriormente discutidos com base na literatura pertinente à temática para melhor apresentação das variáveis.

\section{RESULTADOS E DISCUSSÃO}

\section{Caracterização sociodemográfica dos participantes}

A população estudada foi em sua maioria mulheres, apresentando idade entre 40 e 49 anos, perfazendo um total de $43 \%$, em quanto que um percentual de $15 \%$ dos participantes possui idade igual ou superior a 30 anos. É possível observar a maior prevalência do uso de psicofármacos nas faixas etárias superiores a 30 anos, fato corroborado com Almeida; Coutinho; Pepe (1994), de que o maior consumo de psicofármacos associou-se significativamente com o aumento da idade.

Em relação à situação conjugal dos participantes os dados revelaram que os maiores números de usuários de psicofármacos eram casados, ou seja, $72 \%$, visto que $13 \%$ eram solteiras, $9 \%$ eram divorciados e $6 \%$ viúvos. Diante disso, estudos mostram que indivíduos com companheiros 
e solteiros consumiram significativamente mais psicofármacos que os viúvos e divorciados (RODRIGUES; FACCHINI; LIMA, 2006).

Com relação à escolaridade, $40 \%$ dos entrevistados afirmaram ter o ensino médio completo, $23 \%$ responderam ter concluído o ensino fundamental, $16 \%$ concluíram o ensino superior, $11 \%$ encontram-se cursando ensino superior e $2 \%$ são pós-graduados. Pode se dizer que $69 \%$ da amostra encontra-se em condições vantajosas para entender os riscos / efeitos colaterais dos medicamentos e assimilar as informações oferecidas pelos profissionais de saúde.

Os dados relativos à ocupação revelam que, $43 \%$ dos usuários de psicofármacos são pessoas que desempenham suas atividades domiciliares, $13 \%$ são professores, $11 \%$ comerciantes, $1 \%$ motorista e $14 \%$ não trabalham, isto é, são dependentes de familiares ou são aposentados.

No estudo de Hildebrandt et al., (2009), prevaleceu a profissão de pessoas que desempenham suas atividades domiciliares seguidas pelos aposentados. Em relação aos aposentados demonstram que estão em uma fase da vida, em que em virtude das condições, apresentam altos índices de modificações orgânicas, psíquicas o que, às vezes, gera ansiedade, angústia, medo e sofrimento emocional.

\section{Estilo de vida}

Nos aspectos referentes à ingestão de bebidas alcoólicas, 94\% afirmaram não ingerir nenhum tipo de bebida e $6 \%$ responderam que eventualmente/raramente costuma beber. Sendo que dos $6 \%$ que eventualmente bebem, $4 \%$ bebe a mais de 20 anos, $1 \%$ há 10 anos e $1 \%$ há 9 anos. Observa-se que 51\% relataram já ter bebido alguma vez na vida e $43 \%$ afirmaram nunca ter bebido.

Este questionamento pode ter um viés, já que muitos pacientes ingerem álcool e afirmam que não quando entrevistados, por vergonha ou por saber que a interação álcool x psicofármaco pode ser perigosa.

Acredita-se, que quando bem instruídos sobre a importância da não ingestão de bebidas alcoólicas durante o uso de fármacos depressores do sistema nervoso Central, a terapia torna-se mais eficaz sem possíveis riscos de interações sinérgicas e melhorando a qualidade de vida do paciente.

A ocorrência dessas interações e suas implicações clínicas desperta o interesse na área da psicofarmacologia, já que a prática da polifarmácia é muito freqüiente nesta área, o que se justifica pela dificuldade de diagnóstico das doenças ou distúrbios que as envolvem (OGA; BASILE; CARVALHO, 2002; MIYASAKA; ATALLAH, 2003).

\section{Terapia Medicamentosa}

No gráfico 1, observamos que os ansiolíticos (Benzodiazepínicos) foram os mais consumidos (44\%), seguidos pelos anticonvulsivantes (16\%) e antidepressivos (16\%), $7 \%$ utilizavam opióides, $2 \%$ anorexígenos, $2 \%$ antipsicóticos, $2 \%$ antiparkisoniano, 2\% antialzheimer, e em menor quantidade $1 \%$ usavam anfetaminas e $8 \%$ faziam uso de mais de um psicofármacos.
Gráfico 1 - Distribuição percentual das principais classes de psicofármacos mais utilizados

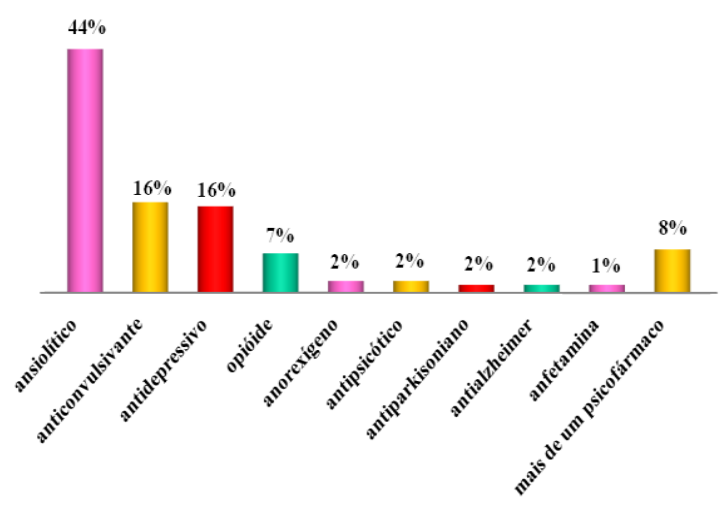

Alguns estudos realizados no Brasil verificaram que a prevalência do consumo de benzodiazepínicos é bastante elevada, quando comparada a outros países; entretanto, assim como na maioria dos estudos conduzidos em outros países, verificou-se que o consumo é mais prevalente nas mulheres e aumenta com a idade (CARLINI, 1995; MARI; ALMEIDA; COUTINHO, 1993; ALMEIDA; COUTINHO; PEPE, 1994).

O estudo também mostrou que o Clonazepam foi o medicamento mais utilizado entre todos os mencionados. Na década de 70, o BZP mais usado era o Diazepam, que nessa época liderou o ranking de medicamentos mais vendidos nos EUA. Atualmente, o BZP mais prescrito é o Clonazepam devido ao seu excelente efeito ansiolítico, hipnótico, relaxante muscular e anticonvulsivante, em contrapartida, este fármaco tem um grande potencial de causar dependência e tolerância (CHARNEY; MIHIC; HARRIS, 2005).

Com relação ao tempo de uso dos psicofarmácos 138 usuários tomaram o medicamento diariamente, isto é, pelo menos uma vez todos os dias. Quanto ao tempo de uso, $40 \%$ utilizavam a mais de um ano; $23 \%$ a um ano; $23 \%$ a seis meses; $7 \%$ a quatro meses; $4 \%$ a mais de dez anos e apenas $3 \%$ a dois meses. Os usuários crônicos corresponderam a $44 \%$. Já com relação a melhora dos sintomas cento e trinta e dois $(94 \%)$ relataram ter tido melhoras após o uso do medicamento e apenas oito $(6 \%)$ afirmaram não apresentar melhoras no seu quadro clínico (Gráfico2).

Gráfico 2 - Distribuição percentual do tempo em que os

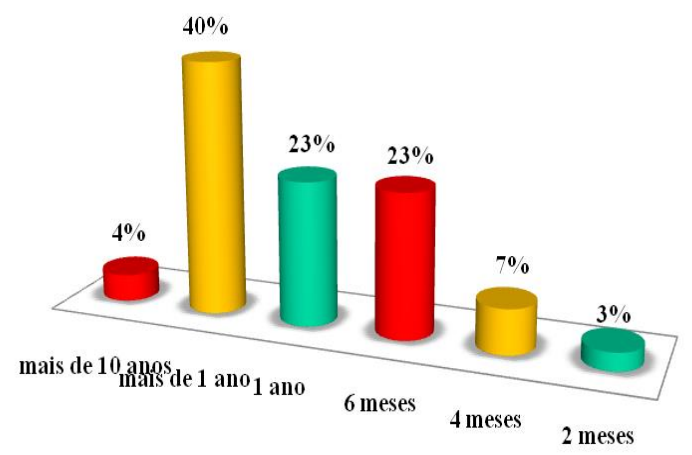

O fato de que $40 \%$ dos pacientes utilizavam por mais de um ano, demontra que a maioria dos 
psicofármacos causam dependência, por isso faz com que os usuários utilizem de maneira crônica.

Nossos dados permitem caracterizar os usuários crônicos como aqueles que tomam um elevado número de medicamentos e que tende a se queixar da persistência da sintomatologia. O número de problemas de saúde mencionados, assim como o número de medicamentos consumidos, é um indicador do estado de saúde do indivíduo.

Tratamento de longa duração expõem o paciente a possíveis interações de importância clínica, pois no decorrer do tratamento podem aparecer diversas condições clínicas que exigem a utilização de medicamentos, e estes devem ser criteriosamente analisados quando prescritos e dispensados (PERUCCA et al., 1994).

Já com relação às principais indicações terapêuticas para o uso dos psicofármacos, verificou-se que o uso dos mesmos seu deu para tratamento da insônia (Gráfico3).

Gráfico 3 - Distribuição percentual das principais indicações terapêuticas dos psicofármacos.

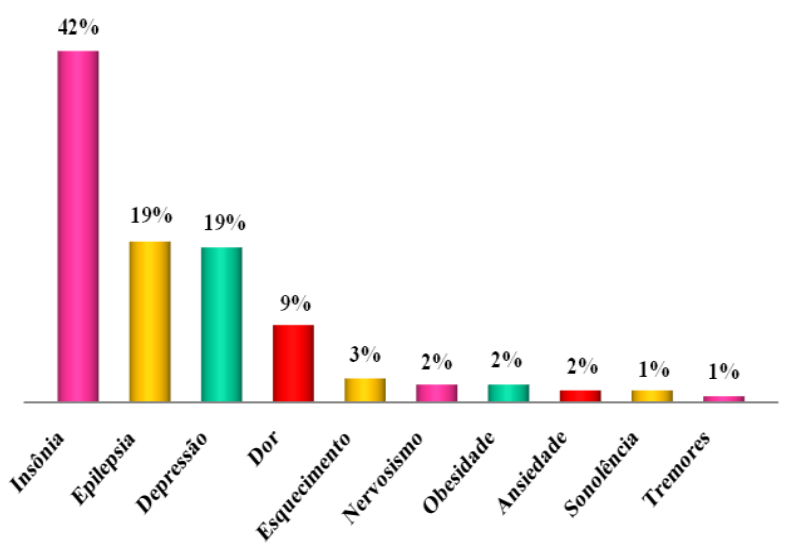

Verifica-se que a maioria dos entrevistados apresenta problemas relacionados ao sono o que para Simon; Vonkorff; Barlow (1996), a queixa de insônia aparece como fator de risco para o uso prolongado de ansiolíticos, o que pode ser considerado um indicador de gravidade e cronicidade, aumentando assim o risco de interações medicamentosas e efeitos adversos, o que torna este grupo de usuários crônicos mais vulnerável.

Corroborando, Tamblyn (1996), salienta que tanto os médicos quanto pacientes relatam que a terapia com os ansiolíticos (benzodiazepínicos) são as mais difíceis de interromper, que $50 \%$ dos pacientes que interrompem o tratamento reiniciaram o uso após um ano.

Dos entrevistados $92 \%$ relataram não ter recebido nenhum tipo de orientação, enquanto $8 \%$ relataram ter recebido algumas orientações, mostrando assim que mesmo em pequena quantidade alguns profissionais médicos estão repassando informações sobre os possíveis riscos desses medicamentos, como mostram as falas transcritas abaixo:

"Não ingerir bebida alcoólica."

"Não tomar anticoncepcional."

"Causa sonolência, náuseas."
"Causa sonolência."

Observou-se que a orientação médica sobre os efeitos colaterais está distante de ser ideal. O fato da orientação mais citada ter sido "não ingerir bebida alcoólica" demonstra que a classe médica confere maior relevância à interação farmacológica, já que a interação álcool x psicofármacos resultante pode ser perigosa, mesmo que o indivíduo utilize álcool socialmente. Este resultado também pode ter sido influenciado pela questão cultural de pacientes e médicos a respeito do conceito popular de incompatibilidade do álcool com medicamentos em geral (AUCHEWSKI, et al., 2004).

Cabe ao profissional farmacêtico, orientar os usuários de psicofármacos durante a atenção farmacêutica, para que o paciente tenha consciência sobre os riscos/efeitos colaterais desses medicamentos. Principalmente quanto às possíveis complicações relacionadas ao uso prolongado do medicamento.

É de extrema importância que o paciente tenha conhecimento dos efeitos colaterais que o uso dos psicofármacos pode levar, pois este conhecimento reduz a falha na adesão ao tratamento. Bem como, é necessário que os usuários saibam dos riscos que esse medicamento pode causar como dependência e tolerância.

Gráfico 4 - Distribuição percentual dos principais efeitos colaterais apresentados pelos usuários de psicofármacos.



Dos usuários de psicofármacos $64 \%$ relataram não apresentar nenhum tipo de efeito colateral e apenas $36 \%$ disseram ter sentido efeitos colaterais, como sonolência $(21 \%)$, dores de cabeça $(8 \%)$, tontura $(4 \%)$ e náuseas (3\%) (gráfico 4).

Muitos dos psicofármacos são depressores do SNC, como os benzodiazepínicos, por isso $21 \%$ dos usuários relataram como efeito colateral sonolência que é um efeito já esperado do uso desse medicamento (RANG et al., 2007).

O impacto do uso de medicamentos em uma sociedade tem várias facetas. Por um lado, os medicamentos podem aumentar a expectativa de vida, erradicar certas doenças, trazer benefícios sociais e econômicos, e por outro lado podem aumentar os custos da atenção à saúde se utilizados inadequadamente e ou levar à ocorrência de reações adversas/efeitos colaterais. (PFAFFENBACH; CARVALHO; MENDES, 2002).

Verificou-se no estudo que apenas $1 \%$ afirmaram ter problemas no convívio familiar após a terapia com psicofármacos, isso demonstra que mesmo fazendo uso de fármacos que alteram as funções neurológicas os pacientes mantiveram-se estáveis. Pois sabe-se que o uso de 
psicofármacos pode até melhorar o convívio familiar, já que o paciente pode reduzir os seus quadros de agitação, nervosismo e insônia. E dessa forma, tratando corretamente essas desordens do SNC, ela pode ter uma vida mais estável, e com até melhoria da sua qualidade e vida.

Gráfico 5 - Distribuição percentual sobre o uso de outras terapias para tratar desordens do sistema nervoso

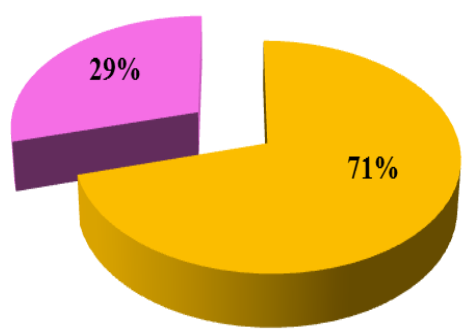

\section{\ão \\ - Fitoterapia}

O gráfico acima demonstra que, $29 \%$ disseram que fazia uso da fitoterapia, como chás, infusões ou medicamentos fitoterápicos e $71 \%$ responderam que não utilizam nenhum outro tipo de terapia para tratar desordens do sistema nervoso.

É importante destacar que o uso de plantas medicinais no tratamento e na cura de enfermidades é bastante antigo, muitas vezes o uso de plantas medicinais é o único recurso terapêutico de muitas comunidades. E atualmente a fitoterapia tem sido utilizada como tratamento padrão ou auxiliar de desordens do SNC, como por exemplo, Valeriana officinalis que tem sido prescrita no tratamento de insônia e ansiedade, além de infusões de camomila e maracujá.

Por meio das observações populares sobre o uso e a eficácia de plantas medicinais, ocorreu a expansão da divulgação das propriedades terapêuticas dos vegetais, o que tem contribuído para a prática do consumo de fitoterápicos, tornando válidas informações terapêuticas que foram acumuladas durante séculos (MACIEL; PINTO; VEIGA, 2002).

De acordo com a Organização Mundial de Saúde (OMS), $80 \%$ da população mundial utiliza plantas medicinais como a principal opção terapêutica, o que representa um fator de grande relevância para a manutenção da saúde. Além da comprovação da ação terapêutica de várias plantas utilizadas popularmente, a fitoterapia representa parte importante da cultura de um povo, sendo também parte de um saber utilizado e difundido pelas populações ao longo de várias gerações (TOMAZONNIZ; NEGRELLE; CENTA, 2006).
Gráfico 6 - Distribuição percentual de outros medicamentos consumidos pelos usuários de psicofármacos

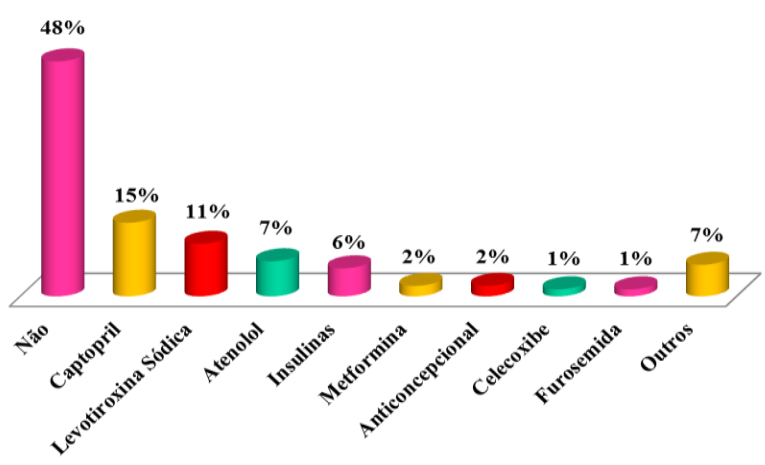

Observou-se que $52 \%$ responderam que fazem uso de outros medicamentos concomitantemente ao tratamento com psicofármacos. Os medicamentos utilizados por esses pacientes apresentam-se expressos em distribuição percentual no gráfico 8 . Pode-se observar que o Captopril e a Levotiroxina Sódica são os principais fármacos utilizados em paralelo com os psicofármacos, mas também foram citados o Atenolol e as Insulinas. Em menor número, foram citados Metformina, Anticoncepcional, Celecoxibe e Furosemida.

A prática da polifarmácia é comum entre os portadores de doenças crônicas, que fazem uso simultâneo de mais de um tipo de medicamento por longos períodos, o que pode resultar em interações medicamentosas.

Uma importante interação farmacológica que podemos citar resulta da interação da carbamazepina, que é um indutor enzimático das isoenzimas CYP (Sistema Enzimático Citocromo P450) com anticoncepcionais, onde o seu uso concomitante pode ocasionar o aumento da metabolização do anticoncepcional, e conseqüentemente dimuindo o seu efeito farmacológico (SANTOS, et al., 2009).

\section{CONCLUSÕES}

Percebe-se que esta havendo um aumento da incidência de transtornos psíquicos em várias partes do mundo, o que é um problema de saúde pública, com uma dimensão crescente e que gera um impacto social e econômico.

As exigências da vida moderna e a forma como cada indivíduo se adapta de modo a poder responder-lhes, pode originar ansiedade, stress, perturbações do sono e sintomas depressivos.

Diante do estudo realizado observou-se que há um aumento progressivo do uso de psicofármacos com a idade, sendo este mais comum entre mulheres, e que os mais utilizados são os ansiolíticos (Clonazepam e Alprazolam), sendo a insônia o principal motivo de uso, a profissão predominante foi as atividades domiciliares, seguida por aqueles que não trabalham (aposentados e dependentes de familiares). Estes dados sugerem à necessidade de políticas públicas e ações de prevenção e promoção de saúde mental direcionadas as mulheres e aos aposentados e de ações intersetoriais que favoreçam a melhoria dos níveis de instrução da população. 
A maioria são pessoas freqüentaram a escola e não ingerem bebidas alcoólicas, assim são bem instruídas e tem condições vantajosas para assimilar as informações oferecidas pelos profissionais de saúde.

Mais da metade dos entrevistados disseram não ter obtido orientação médica sobre os riscos que os psicofármacos podem acarretar. Os entrevistados que foram mais bem orientados ao receberem uma prescrição de psicofármacos tiveram maior percepção da melhora do seu quadro, conclui-se que há necessidade dos profissionais de saúde informarem aos seus pacientes os riscos/efeitos colaterais que esse fármacos podem causar. Daí a importância também do profissional farmacêutico de fazer a assistência farmacêutica no momento da dispensação para aumentar a adesão ao tratamento e reduzir os efeitos colaterais e interações farmacológicas.

Houve prevalência do uso diário dos psicofármacos e por mais de um ano, verifica-se também o uso da fitoterapia como uma terapia alternativa para tratar sintomas como a insônia e ansiedade, e de outros medicamentos o que pode ser um indicativo de pessoas que possui doenças crônicas e que pode gerar interações medicamentosas.

A epidemiologia do uso de psicofármacos entre os indivíduos atendidos em uma farmácia comunitária no município de Cajazeiras - PB, em geral segue os dados de outros estudos relatados na literatura cientifica.

$\mathrm{E}$ os resultados obtidos neste estudo são de extrema importância, pois mostra que há necessidade de maior orientação dos pacientes com relação ao uso desses medicamentos. E dentro desse contexto, o farmacêutico tem um papel importantíssimo para orientar esses pacientes, a fim de evitar efeitos tóxicos, interações medicamentosas e aumentar a eficácia do tratamento, tentando reduzir as falhas na adesão ao tratamento com psicofármacos.

Este estudo também nos mostra que a prescrição de BZP esta sendo crescente e muitas vezes irracional, pois muitos pacientes com transtornos psíquicos podem ser tratados com medicamentos fitoterápicos que tem menos efeitos colaterais e que não causam dependência e tolerância, ou até mesmo com terapias não farmacológicas, como acupuntura, exercício físico, hidroginástica ou psicoterapia.

\section{REFERÊNCIAS BIBLIOGRÁFICAS}

ALVES, D.S.N. Reestruturação da atenção em saúde mental: situação atual: diretrizes e estratégias. In: AMARANTE, P. Psiquiatria social e reforma psiquiátrica. Rio de Janeiro: Fiocruz, 1994. p. 197- 204.

ALMEIDA, L.M; COUTINHO E.S.F; PEPE V.L.E. Consumo de Psicofármacos em uma Região Administrativa do Rio de Janeiro: A Ilha do Governador. Cadernos de Saúde Pública, Rio de Janeiro, v.10, n.1, p.516 jan./mar.1994. Disponível em: <http://www.scielo.br>. Acesso em: 07 de Fev. 2011.

AUCHEWSKI, L.; ANDREATINI, R.; GALDURÓZ, J.C.F.; LACERDA, R.B. Avaliação da orientação médica sobre os efeitos colaterais de benzodiazepínicos. Revista Brasileira de Psiquiatria. v. 26, n. 1, p.24-31, 2004.
Disponível em: <http://www.scielo.br>. Acesso em: 22 de Out. 2011.

BRASIL, M.S. Ministério do Planejamento, Orçamento e Gestão. Instituto Brasileiro de Geografia e Estatística, Brasília, 2010.

CARLINI, E.A. Benzodiazepínicos no Brasil: Um perfil de consumo nos anos de 1988 a 1989. In: CARLINI, E.A. (org.). Medicamentos, Drogas e Saúde. São Paulo: Hucitec/Sobravime, 1995. p.119-129.

CHARNEY, D. S; MIHIC, S. J; HARRIS, R. A. Hipnóticos e Sedativos. In: GILMAN, A.G.(editorconsultor). As Bases Farmacológicas da Terapêutica. 10 ed. Rio de Janeiro: Mc Graw Hill, 2005 p. 301-324.

DUNCAN, B.B; SCHMIDT, M.I; GIUGLIANI, E.R.J. Medicina ambulatorial: Condutas de

atenção primária baseadas em evidências. 3 ed. Porto Alegre: Artmed, 2004.

HILDEBRANDT, L.M; LEITE, L.M; PIOVESAN, S.M.S; STUMM, L.K. Prevalência no consume de psicotrópicos pela população assistida por um serviço municipal se saúde. Disponível em: <http://www.madres. org/asp/contenidoscielo.br. Acesso: 14 de Out. de 2011.

HILDEBRANDT, L.M; LEITE, L.M; PIOVESAN, S.M.S; STUMM, L.K. Prevalência no consume de psicotrópicos pela população assistida por um serviço municipal se saúde. Disponível em: <http://www.madres. org/asp/contenidoscielo.br. Acesso: 14 de Out. de 2011.

NOTO, A.R.; CARLINI, E.A.; MASTROIANNI, P.C.; ALVES, V.C.; GALDURÓZ, J.C.F.; KUROIWA, W.; CSIZMAR, J.; COSTA, A.; FARIA, M.A.; HIDALGO, S.R.; ASSIS, D.; NAPPO, S.A.. Análise da prescrição e dispensação de medicamentos psicotrópicos em dois municípios do Estado de São Paulo. Revista Brasileira de Psiquiatria, São Paulo, v.24, n.2, p. 68-73, 2002. Disponível em: <http://www.scielo.br>. Acesso em: $15 \mathrm{de}$ Mar. 2011.

RANG, H.P.; DALE, M.M.; RITTER, J.M.; FLOWER, R.J. Farmacologia. 6 ed. Rio de Janeiro: GuanabaraKoogan, 2007.

RODRIGUES, M. A; FACCHINI, L. A; LIMA, M. S. Modificações nos padrões de consumo de psicofármacos em localidade do Sul do Brasil. Revista de Saúde Pública, São Paulo, v.40, n. 1, p.107-114, jan./fev. 2006. Disponível em: <http://www.scielo.br>. Acesso em: $14 \mathrm{de}$ Mar. 2011.

SANTOS, H.C.; RIBEIRO, R.R.; FERRANI, M.; FERNANDES, J.P.S. Possíveis interações medicamentosas com psicotrópicos encontradas em pacientes da zona leste de São Paulo. Revista Ciências Farmacêuticas Básica Aplicada, São Paulo, v. 30, n. 3, p. 285-289, 2009. 
SIMON, G.E; VONKORFF, M.; BARLOW, W. Predictors of chronic benzodiazepine use in a health maintenance organization sample. Journal of Clinical Epidemiology, v.49, p.1067-1073, 1996. Disponível em: <http://www.scielo.br>. Acesso em: 24 de Out. 2011.

MACIEL, M.A.M.; PINTO, A.C.; VEIGA, V.F.J. Plantas medicinais: A necessidade de estudos multidisciplinares. Química Nova. Rio de Janeiro, v.25, n.3, p.429-438, 2002. Disponível em: <http://www.scielo.br〉. Acesso em: 22 de Out. 2011.

OGA, S.; BASILE, A.C.; CARVALHO, M.F. Guia Zanini-Oga de interações medicamentosas. São Paulo: Atheneu, 2002.

PERUCCA, E.; GATTI, G.; CIPOLLA, G.; SPINA, E.; BAREL, S.; SOBACK, S. Inhibition of diazepam metabolism by fluvoxamine: a pharmacokinetic study in normal volunteers. Clinical Pharmacology and Therapeutics, v.56, n. 5, p.471-476, 1994. Disponível em: <http://www.scielo.br>. Acesso em: 24 de Out. 2011.

PFAFFENBACH, G.; CARVALHO, O.M.; MENDES, G.B. Reações adversas a medicamentos como determinantes da admissão hospitalar. Revista Associação médica Brasileira. São Paulo, v. 48, n.3, p.237-241, 2002. Disponível em: <http://www.scielo.br>. Acesso em: 25 de Out. 2011

TOMAZZONIZ, M.I.; NEGRELLE, R.R.B.; CENTA, M.L. Fitoterapia Popular: A busca instrumental enquanto prática terapêutica. Texto Contexto Enfermagem, v.15, n.1, p.115-121, 2006. Disponível em: <http://www.scielo.br>. Acesso em: 24 de Out. 2011.

TAMBLYN, R.N. Medication on use in seniors: challenges and solutions. Thérapie, v. 51, n. 3, p. 269-282, mai./jun. 1996. Disponível em: <http://www.scielo.br>. Acesso em: 24 de Out. 2011. 